\section{Stop the presses}

A self-cleaving degradation tag allows researchers to quickly and reversibly shut down protein production.

There are many ways to temporarily switch a gene off-one can inactivate a conditionally expressed gene, for example, or prevent protein production via RNA interference (RNAi). However, these approaches often fail to efficiently deplete the target protein, and they typically result in considerable delays before depletion can be fully enacted or reversed.

Michael Lin and colleagues at Stanford University have now devised a highly effective alternative: a 'self-destruct' tag for proteins that automatically removes itself unless it is inhibited with a cell-permeable, nontoxic drug. Their small molecule-assisted shutoff (SMASh) tag comprises a degradation signal and a protease enzyme from hepatitis $\mathrm{C}$ virus (HCV), which are attached to the target protein through a sequence that serves as a substrate for the protease. This tag promptly cleaves itself after translation, leaving behind an intact and minimally modified target protein. But in the presence of asunaprevir, a clinically approved HCV protease inhibitor, the tag remains attached and the whole construct is promptly degraded.

The researchers demonstrated that they could swiftly terminate the production of SMASh-tagged proteins with relatively low levels of asunaprevir. For example, $1.5 \mu \mathrm{M}$ asunaprevir proved sufficient to abruptly terminate new expression of labeled yellow fluorescent protein (YFP) in mammalian cells; because already-translated protein molecules remain intact, this also offers a useful means for studying protein half-lives. The effect is reversible as well: within an hour after the drug had been washed away, cells had already synthesized new YFP molecules. This is in contrast to strategies such as RNAi, the effects of which can take days to reverse.
Asunaprevir efficiently penetrated mammalian cell membranes, but it was also capable of crossing cell walls to achieve SMASh-tag inactivation in yeast experiments. And because the drug has already been tested for safety in humans, a SMASh-style approach could potentially be used clinically to control the activity of cell-, virus- or foreign gene-based therapies. As proof of concept, Lin and colleagues demonstrated that their technique could be used to selectively inactivate a viral agent currently being tested for cancer therapy, using doses of asunaprevir that are known to be nontoxic to patients. Michael Eisenstein

\section{RESEARCH PAPERS}

Chung, H.K. et al. Tunable and reversible drug control of protein production via a self-excising degron. Nat. Chem. Biol.

doi:10.1038/nchembio.1869 (27 July 2015). 